\title{
Random-gate-voltage induced Al'tshuler-Aronov-Spivak effect in topological edge states
}

\author{
Kun Luo, ${ }^{1,2}$ Wei Chen, ${ }^{1,2, \text { f }}$ Li Sheng, ${ }^{1,2}$ and D. Y. Xing ${ }^{1,2}$ \\ ${ }^{1}$ National Laboratory of Solid State Microstructures and department of Physics, Nanjing University, Nanjing, 210093, China \\ ${ }^{2}$ Collaborative Innovation Center of Advanced Microstructures, Nanjing University, Nanjing 210093, China
}

(Dated: November 19, 2021)

\begin{abstract}
Helical edge states are the hallmark of the quantum spin Hall insulator. Recently, several experiments have observed transport signatures contributed by trivial edge states, making it difficult to distinguish between the topologically trivial and nontrivial phases. Here, we show that helical edge states can be identified by the random-gate-voltage induced $\Phi_{0} / 2$-period oscillation of the averaged electron return probability in the interferometer constructed by the edge states. The random gate voltage can highlight the $\Phi_{0} / 2$-period Al'tshuler-Aronov-Spivak oscillation proportional to $\sin ^{2}\left(2 \pi \Phi / \Phi_{0}\right)$ by quenching the $\Phi_{0}$-period Aharonov-Bohm oscillation. It is found that the helical spin texture induced $\pi$ Berry phase is key to such weak antilocalization behavior with zero return probability at $\Phi=0$. In contrast, the oscillation for the trivial edge states may exhibit either weak localization or antilocalization depending on the strength of the spin-orbit coupling, which have finite return probability at $\Phi=0$. Our results provide an effective way for the identification of the helical edge states. The predicted signature is stabilized by the time-reversal symmetry so that it is robust against disorder and does not require any fine adjustment of system.
\end{abstract}

\section{INTRODUCTION}

Over the past decade, topological phases of matter such as topological insulator and superconductor have become a new research field of condensed matter physics [1, 2]. As the first theoretically proposed and experimentally implemented topological material, the quantum spin Hall insulator (QSHI) has attracted broad research interest [3-10]. The fingerprint of QSHI is the existence of topologically protected helical edge states at the sample boundary [3, 5]. Due to spin-momentum locking, helical edge states provide an interesting platform to study exotic electronic properties. Moreover, such strongly spin-orbit coupled system can also lead to important applications such as spintronic device, lowconsumption transistor [1, 2] and topological quantum computation [11-13].

Although the edge states have been confirmed by measuring the edge conductance in various QSHI materials [7-10], its helical nature remains to be identified. Recently, topologically trivial edge states induced by band bending have been observed in various materials, such as InAs/GaSb quantum wells [14 17, InAs [16, 18] and $\mathrm{InSb}$ [19. These trivial edge states result in similar transport signatures which make it difficult to discriminate them from the helical ones. Several theoretical proposals have been put forward for the detection of helical spin texture of the edge states [20 26], yet their implementation remains challenging, which are either beyond state-of-the-art fabrication technique or requires a fine tuning of the sample parameters. Therefore, it is highly demanded to find a clear and robust signature to discriminate the helical edge states from the trivial ones without

* Corresponding author: pchenweis@gmail.com (a)

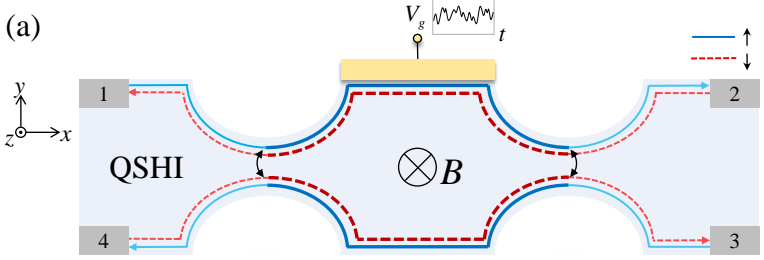

(b)

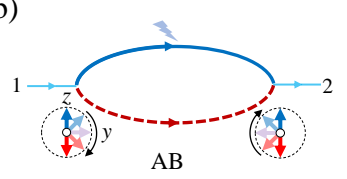

(c)

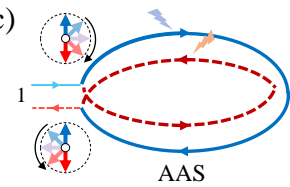

FIG. 1. (a) The interferometer constructed by the edge states of the QSHI. The spin up (down) edge states are denoted as blue solid (red dashed) lines, with their moving directions labeled by arrows. Two point contact structures allow the coupling between the upper and lower edge states. A side gate (yellow bar) with random voltage $V_{g}$ is deposited at the upper edge to introduce dephasing effect. Weak magnetic field $B$ is imposed perpendicular to the sample. Four terminals (14) are sketched as gray bars. (b) The AB interfering loop is fragile to the random gate. The spin flipping accompanies the inter-edge transmission. (c) AAS interfering loop is immune to the random gate. The $\pi$ Berry phase associated with the spin rotation gives rise to zero return probability.

fine tuning of the system.

In this work, we investigate coherent electron transport through an Aharonov-Bohm (AB) interferometer composed of edge states; see Fig. 11(a). By imposing a random gate to the interferometer, the main $\Phi_{0}$-period $\mathrm{AB}$ oscillation is suppressed due to the dephasing effect [cf. Fig. 1(b)], giving rise to a dominant Al'tshuldrAronov-Spivak (AAS) oscillation with $\Phi_{0} / 2$ periodicity [cf. Fig. 11(c)]. Different from the conventional AAS oscillation, here, the weak antilocalization effect is in- 
duced by the random gate rather than disorder averaging [27, 28]. The results are generally stabilized by the time-reversal symmetry, thus offering a robust signature of the helical spin texture that does not rely much on the sample details. Specifically, the AAS oscillation of the return probability takes the form $\bar{R}=\mathcal{R} \sin ^{2}\left(2 \pi \Phi / \Phi_{0}\right)$. The absence of return probability at $\Phi=0$ is attributed to the destructive AAS interference induced by the $\pi$ spin Berry phase accumulated during inter-edge tunneling, a direct result of the helical spin texture. For the trivial edge state, the return probability take the general form $\bar{R}= \pm \mathcal{R} \sin ^{2}\left(2 \pi \Phi / \Phi_{0}\right)+\mathcal{R}_{0}$ with $\mathcal{R}_{0}>0$, where the " \pm " is determined by the strength of the spin-orbit coupling. In contrast to the helical edge states, nonzero return probability occurs at $\Phi=0$ for the trivial edge states due to the doubling of transport channels. The different oscillation patterns of the return probability can be measured by the differential conductance. Therefore, our scheme provides an unambiguous evidence to discriminate between the nontrivial and trivial edge states.

The rest of this paper is organized as follows. In Sec. III we explicate the basic physical picture of the main results. In Sec. III] we adopt a scattering matrix analysis based on the effective model of the edge states to solve the random gate induced $\Phi_{0} / 2$-period oscillation of the return probability. Detailed numerical simulations on the lattice model is conducted in Sec. IV to show the robustness of the main results for the helical edge states. In Sec. V] we study the AAS oscillation for the trivial edge states and compare the results with that of the helical edge states. Finally, a brief summary and discussion are given in Sec. VI

\section{PHYSICAL PICTURE}

The AB interferometer constructed by the edge states of the QSHI is illustrated in Fig. 1(a), where the spin up (down) edge states propagate in the clockwise (anticlockwise) direction. Two point contacts (PCs) are fabricated to introduce inter-edge coupling [29, such that the central region of the setup forms a closed interfering loop. A weak magnetic field is applied, which acts on the electron motion through the flux modulation $\Phi=B S$ in the area $S$ enclosed by the interference loop. At two PCs, Rashba spin-orbit coupling can be induced by local electric fields [29 32, which gives rise to inter-edge transmission, i.e., electrons in one edge transmit to the opposite edge with the same moving direction accompanied by a spin flipping [26, 32. Here, we apply a gate voltage $V_{g}$ to the upper edge; see Fig. 1(a), which introduces an additional phase factor $\delta=e V_{g} L / v$ to the wave function of the upper electron, with $L$ the length of the gate and $v$ the velocity of the edge states. Here, $V_{g}$ takes random values, which is key to the main results of this work.

Consider an electron propagates coherently in the $\mathrm{AB}$ interferometer, which contains multiple interfering loops. Two typical ones among them are the main $\mathrm{AB}$ and $\mathrm{AAS}$ interfering loops, as shown in Figs. 1(b) and 11(c), respectively. The main AB loop is composed of the upper and lower transmission channels, which results in a $\Phi_{0}$-period oscillation of transmission probability. Such interference is sensitive to the phase shift $\delta$ induced by $V_{g}$ so that a random $V_{g}$ causes dephasing effect and quenches such $\mathrm{AB}$ oscillation. In contrast, the AAS interference consists of two backscattering trajectories being the time-reversal counterpart to each other. As the time scale of $V_{g}$ fluctuation is much larger than that an electron spends inside the interferometer, the pair of paths gain the same random phase $\delta$. Therefore, such AAS interference survives, giving rise to a dominant $\Phi_{0} / 2$-period oscillation of the return probability.

Importantly, the electron return probability contains the key information of the helical spin texture of the edge states. Consider an electron incident from terminal 1 in Fig. 1(a), both two backscattering trajectories involves an inter-edge transmission along with a $\pi$ spin rotation about the $x$ axis in opposite directions denoted by the rotation operator $O_{x}( \pm \pi)$; see Fig. 1(c). Accordingly, the backscattering wave function takes the form $r_{0} e^{i \delta}\left[O_{x}(-\pi)+e^{i 4 \pi \Phi / \Phi_{0}} O_{x}(\pi)\right]|\uparrow\rangle$, with $r_{0}$ the backscattering amplitude for each path. It reduces to $r_{0} e^{i \delta}\left[1+e^{i 4 \pi \Phi / \Phi_{0}} O_{x}(2 \pi)\right]|\downarrow\rangle$ and gives rise to the probability $\propto \sin ^{2}\left(2 \pi \Phi / \Phi_{0}\right)$ using the property of the spin Berry phase $O_{x}(2 \pi)=e^{i \pi}$. Since the spin rotation is enforced by the spin-momentum locking, such an oscillation behavior directly reveals the spin texture of the edge states. For zero magnetic field with $\Phi=0$, the return probability vanishes, which is prohibited by the time-reversal symmetry of the helical edge states. A small magnetic flux breaks the time-reversal symmetry and increases the return probability, indicating a weak antilocalization effect.

\section{SCATTERING MATRIX ANALYSIS}

In this section, we study electron transport in the interferometer using the scattering matrix approach based on the low-energy model of the edge states. The scattering matrix of the whole interferometer is obtained by combining the scattering matrices at two PCs and involving the phase accumulation during electron propagation. The scattering matrix at the left PC can be parameterized as

$$
S_{L}=\left(\begin{array}{cccc}
0 & r_{1} & -t_{1} & -t_{2} \\
-r_{1} & 0 & -t_{2} & -t_{1} \\
t_{1} & t_{2} & 0 & r_{2} \\
t_{2} & t_{1} & -r_{2} & 0
\end{array}\right)
$$

which relates the incident and outgoing waves via $b_{L}=$ $S_{L} a_{L}$. The four components of the wave $a_{L}, b_{L}$ correspond to the upper and lower channels on the left and right side of the PC. Here, the antisymmetric condition $S_{L}^{T}=-S_{L}$ is taken into account due to the time-reversal symmetry of the helical states 33. The amplitudes $t_{1}, t_{2}$ 
are the intra- and inter-edge transmission, respectively; $r_{1}, r_{2}$ are the inter-edge reflection on both sides of the PC, while the intra-edge reflection with spin flipping is prohibited by the time-reversal symmetry. The matrix for the right $\mathrm{PC} S_{R}$ is defined in the same way. The phase modulation of the interferometer comes from the gate voltage $V_{g}$ deposited on the upper channel [cf. Fig. [1(a)] and the flux $\Phi$ in the middle area encircled by the interfering loop, which can be described by the matrix

$$
S_{M}=\left(\begin{array}{cccc}
0 & 0 & e^{i\left(\delta-\phi_{1}\right)} & 0 \\
0 & 0 & 0 & e^{i \phi_{2}} \\
e^{i\left(\delta+\phi_{1}\right)} & 0 & 0 & 0 \\
0 & e^{-i \phi_{2}} & 0 & 0
\end{array}\right)
$$

where $\phi_{1,2}$ are the extra phase due to the magnetic field, with the sum $\phi=\phi_{1}+\phi_{2}=2 \pi \Phi / \Phi_{0}$ being gauge invariant.

Combining three matrices $S_{L}, S_{M}, S_{R}$ yields the scattering matrix for the whole interferometer. In the following, $S_{R}=S_{L}$ is adopted for simplicity, which will not change the main results. For an electron injected from terminal 1 , its return probability $R$ and transmission probability $T$ to terminal 2 are obtained after some algebra as

$$
\begin{aligned}
R= & F^{-1} 4\left|r_{1} t_{1} t_{2}\right|^{2} \sin ^{2} \phi \\
T= & F^{-1}\left[|X|^{2}+|Y|^{2}+|Z|^{2}+2|X Y| \cos \left(\phi-\delta+\theta_{x y}\right)\right. \\
& \left.+2|X Z| \cos \left(\phi+\delta+\theta_{x z}\right)+2|Y Z| \cos \left(2 \delta+\theta_{y z}\right)\right] \\
F= & 1+K^{4}+2 K^{2}\left[\cos 2(\delta+\nu)+2 \cos ^{2} \phi\right] \\
& +4 K\left(1+K^{2}\right) \cos \phi \cos (\delta+\nu),
\end{aligned}
$$

where $X=t_{1}^{2}+t_{2}^{2} r_{1} r_{2}, Y=t_{1}^{2} r_{1} r_{2}, Z=t_{2}^{2}, \theta_{i j}=\theta_{i}-\theta_{j}$ with $\theta_{i=x, y, z}$ are the arguments of $X, Y, Z$, respectively, $K=\left|r_{1} r_{2}\right|$ and $\nu=\arg \left(r_{1} r_{2}\right)$.

The scattering probabilities in Eq. (3) contains the information of the competition between the AB and AAS effects. For a fixed $V_{g}$ or equivalently, the phase factor $\delta$, the transmission $T$ is dominated by the $\mathrm{AB}$ oscillation with $\Phi_{0}$ periodicity. Due to the current conservation, the $\Phi_{0}$-period component also appears in the oscillating pattern of the return probability $R$. As $\delta$ takes random values or equivalently, it is integrated out, the first-order AB oscillation quenches and the $\Phi_{0} / 2$-period AAS oscillation dominates the transport. This fact can be clearly seen in the weak reflection limit of the PCs, that is $K \ll 1$. By expanding $F$ in Eq. (3) to the first order of $K$, the return probability reduces to

$$
R \simeq 4\left|r_{1} t_{1} t_{2}\right|^{2} \sin ^{2} \phi[1-4 K \cos \phi \cos (\delta+\nu)],
$$

which contains both $\Phi_{0}$ and $\Phi_{0} / 2$ periodicity. To see the effect due to the random gate voltage, we integrate out $\delta$, which yields

$$
\bar{R} \simeq 4\left|r_{1} t_{1} t_{2}\right|^{2} \sin ^{2} \phi
$$

where only the $\Phi_{0} / 2$-period oscillation remains. Similarly, the averaged transmission probability reduces to

$$
\begin{aligned}
\bar{T} & =|X|^{2}+|Y|^{2}+|Z|^{2} \\
& +2 K|X Y|\left[\cos \left(2 \phi+\theta_{x y}+\nu\right)+\cos \left(\theta_{x y}+\nu\right)\right] \\
& +2 K|X Z|\left[\cos \left(2 \phi+\theta_{x z}-\nu\right)+\cos \left(\theta_{x z}-\nu\right)\right],
\end{aligned}
$$

in which only the $\Phi_{0} / 2$-period oscillation remains, the same as $\bar{R}$. The random $V_{g}$ introduces dephasing effect to the $\mathrm{AB}$ interference process while retains phase coherence between paired AAS interference loops. As a result, the AAS effect dominates the transport, giving rise to the $\Phi_{0} / 2$-period oscillation.

The conclusion above generally holds beyond the weak reflection limit. We provide numerical results of Eq. (3) for a general case in Fig. 2, The return and transmission probabilities for fixed and random $V_{g}$ are shown in Figs. 2 (a) and 2(b), respectively. For a certain $\delta$, the transmission $T$ is dominated by the $\mathrm{AB}$ oscillation with $\Phi_{0}$ periodicity. As $\delta$ is averaged out, both $\bar{T}$ and $\bar{R}$ oscillate with $\Phi_{0} / 2$ periodicity, which is a clear signature of the AAS scenario. The transition of the oscillation period from $\Phi_{0}$ (in $T$ ) to $\Phi_{0} / 2$ (in $\bar{T}$ ) visibly manifests the random gate induced AAS interference. Importantly, here the return probability takes the form of $\bar{R} \propto \sin ^{2} \phi$ which vanishes for $\Phi=0$. It is a direct result of the spin-momentum locking as elucidated in Sec. II thus providing a direct evidence of helical edge states.

\section{LATTICE MODEL SIMULATION}

Based on the scattering matrix analysis, one can see that only the AAS oscillation is stable for a random $V_{g}$. Next, we perform numerical simulation to show the robustness of the results. We adopt the Bernevig-HughesZhang model to describe the QSHI [5]

$$
H_{\mathrm{BHZ}}=-D k^{2}+A k_{x} \tau_{x} \sigma_{z}-A k_{y} \tau_{y}+\left(M-B k^{2}\right) \tau_{z},
$$

where $\sigma_{x, y, z}$ and $\tau_{x, y, z}$ are Pauli matrices acting on the spin and orbital space, respectively. Here, $k^{2}=k_{x}^{2}+k_{y}^{2}$, and $A, B, D, M$ are the material parameters. At the PC, the Rashba spin-orbit coupling is described by $H_{R}=$ $\alpha\left(1+\tau_{z}\right)\left(k_{x} \sigma_{y}-k_{y} \sigma_{x}\right)$ [30]. We map the effective model onto the square lattice through the substitutions $k^{2}=2 a^{-1}\left[2-\cos \left(k_{x} a\right)-\cos \left(k_{y} a\right)\right], k_{x, y}=a^{-1} \sin \left(k_{x, y} a\right)$ with $a$ the lattice constant (see Appendix A for details) and calculate the scattering probabilities using KWANT package 34 .

The geometric parameters of the setup are provided in the caption of Fig. 2 and Appendix A. The side gate voltage $V_{g}$ is applied on the upper edge states, specifically, in the area $3 W / 8<y<W / 2$ of the middle region. The strengths of Rashba spin-orbit coupling around two PCs $\alpha_{L, R}$ are set to an equal value. Consider an electron injected from terminal 1 with an energy within the bulk gap of the QSHI, only the edge channels are available 

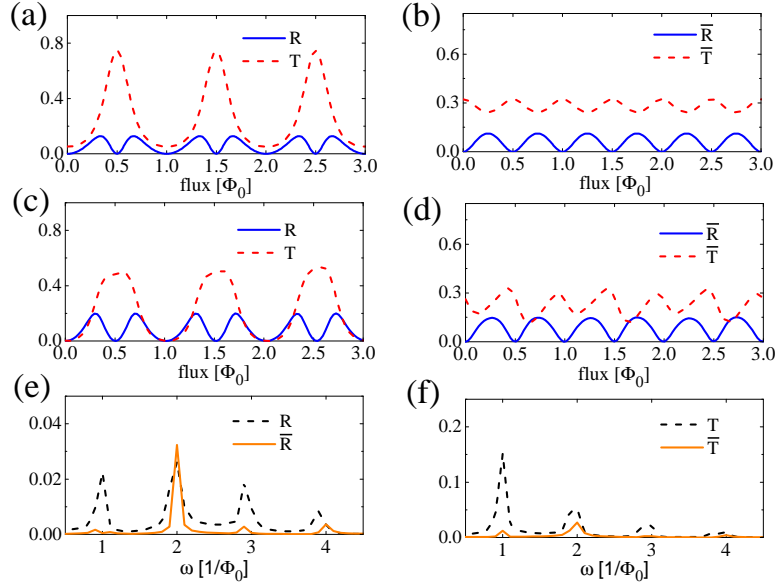

FIG. 2. The oscillation pattern of $R, T(\bar{R}, \bar{T})$ from the edge state analysis for (a) $\delta=0$ and (b) random $\delta$. The matrix elements in Eq. (1) are set as $r_{1}=\sqrt{0.3}, t_{1}=\sqrt{0.5} i$ and $t_{2}=\sqrt{0.2}$. The results of the lattice simulation for (c) $V_{g}=0$ and (d) random $V_{g}$ with $\mathcal{W}=10 \mathrm{meV}$ and an incident energy of $1.5 \mathrm{meV}$. The FFT spectrum of (e) the return probability $R(\bar{R})$ and (f) the transmission probability $T(\bar{T})$. The model parameters are set to $A=364.5 \mathrm{~nm} \mathrm{meV,} B=-686 \mathrm{~nm}^{2}$ $\mathrm{meV}, D=-512 \mathrm{~nm}^{2} \mathrm{meV} M=-10 \mathrm{meV}$. The Rashba strength at both point contacts is $\alpha_{L}=\alpha_{R}=160 \mathrm{~nm} \mathrm{meV}$. The geometric parameters of the system [cf. Appendix A] are set as follows: the width of QSHI sample in the $y$ direction is $W=300 \mathrm{~nm}$, the width of two PCs is $W_{P C}=40 \mathrm{~nm}$, the length of two PCs is $L_{P C}=210 \mathrm{~nm}$ and the length of middle region in between is $L_{M}=180 \mathrm{~nm}$. The results $\bar{R}$ and $\bar{T}$ are averaged over 100 random values of $V_{g}$.

for propagation. The scattering probabilities for $V_{g}=0$ is shown in Fig. 2(c), which resemble those from the edge state analysis in Fig. 2(a). Specifically, $T$ is dominated by the $\Phi_{0}$-period oscillation and $R$ contains multiple periods of oscillation. Then we consider a random $V_{g}$ with uniform distribution $V_{g} \in[0, \mathcal{W}] \mathrm{meV}$. The averaged scattering probability $\bar{T}$ and $\bar{R}$ are shown in Fig. 2 (d). Both $\bar{R}$ and $\bar{T}$ exhibit a dominant $\Phi_{0} / 2$-period oscillation, which manifests a random gate induced AAS effect. Note that $R(\bar{R})$ vanishes for $\Phi=0$, indicates the helical spin texture of the edge states. The transition of the oscillating period is more clearly revealed by the frequency distribution of the oscillating pattern in Figs. 2(e) and 2(f). One can see that there are three peaks at $1 / \phi_{0}, 2 / \phi_{0}, 3 / \phi_{0}$ in the FFT spectra of $R$ with nearly equal amplitude for a fixed $V_{g}$. The random $V_{g}$ enhances the $2 / \phi_{0}$ component in the FFT spectra of $\bar{R}$ while quenches other frequencies, corresponding to a $\Phi_{0} / 2$-period AAS oscillation. For the transmission $T$, it is initially dominated by the $1 / \phi_{0}$ frequency corresponding to the $\Phi_{0}$-period $\mathrm{AB}$ oscillation. After random $V_{g}$ averaging, the AB oscillation of $\bar{T}$ quenches, while the $\Phi_{0} / 2$-period AAS frequency maintains and dominates the transport.
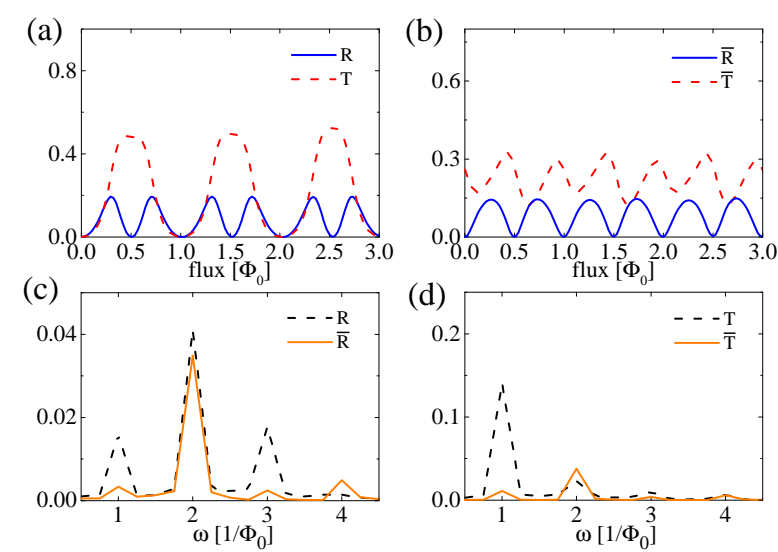

FIG. 3. Oscillation patterns of scattering probabilities by lattice simulation for (a) $V_{g}=0$ and (b) random $V_{g}$ with $\mathcal{W}=$ $10 \mathrm{meV}$. The FFT spectrum of (c) the return probability $R(\bar{R})$ and (d) the transmission probability $T(\bar{T})$. All parameters are the same as those in Fig. 2 except for $\alpha_{R}=160 \mathrm{~nm} \mathrm{meV}$.
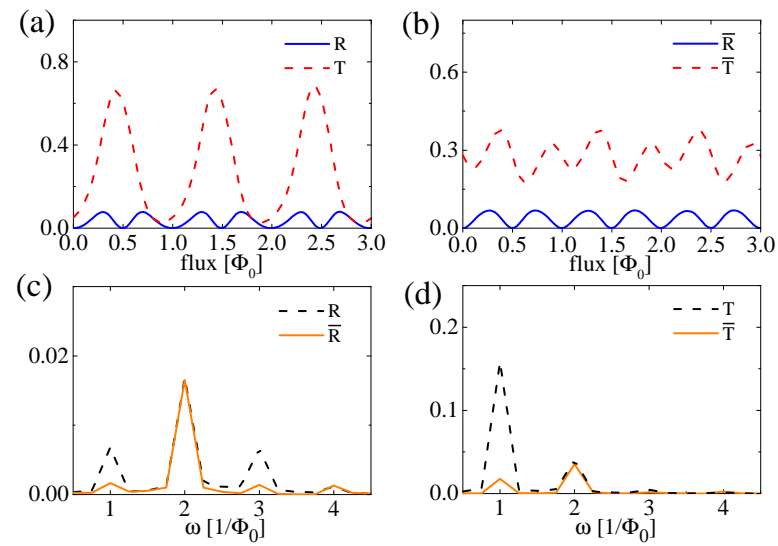

FIG. 4. Oscillation patterns of scattering probabilities and their FFT spectra. All parameters are the same as those in Fig. 2 except for $L_{R P C}=160 \mathrm{~nm}$.

Next we show that the random gate induced AAS effect generally holds for different systemic parameters and is robust against nonmagnetic disorder. First, we change the Rashba coefficient $\alpha_{R}$ at the right PC. The scattering probabilities $T, R(\bar{T}, \bar{R})$ without (with) $V_{g}$ averaging are shown in Fig. 3. One can see that the main results still hold, i.e., the random $V_{g}$ quenches the $\Phi_{0}$-period oscillation and leads to a dominant $\Phi_{0} / 2$-period oscillation as shown in Figs. 3(a) and 3(b). There are three peaks in the frequency domain of $R$, and only a single peak at $2 / \phi_{0}$ survives in $\bar{R}$ after $V_{g}$ averaging; see Fig. 3(c). Accordingly, the $\Phi_{0} / 2$-period AAS oscillation overwhelms the $\Phi_{0}$-period $\mathrm{AB}$ oscillation in the transmission probability $\bar{T}$ as shown in Fig. 3 (d). Similar results also hold as one varies the length $L_{R P C}$ of the right $\mathrm{PC}$ as shown in Fig. 4. Fig. 5 shows the similar results with a different inci- 

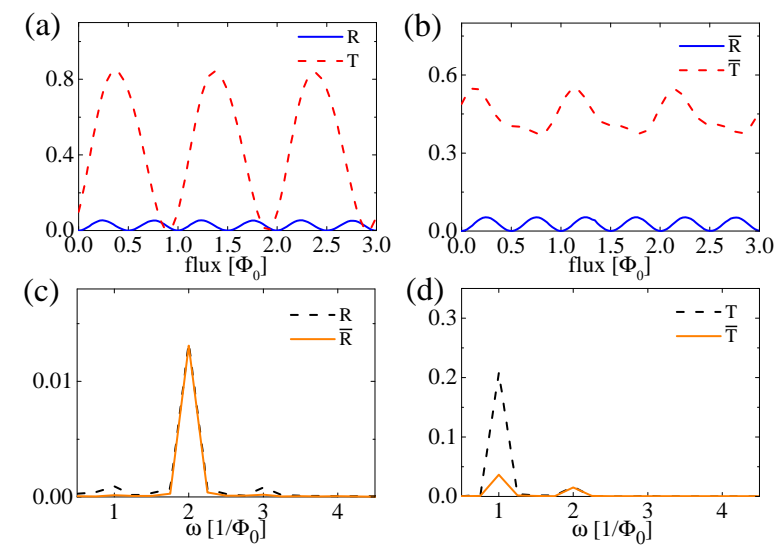

FIG. 5. Oscillation patterns of scattering probabilities and their FFT spectra. All parameters are the same as those in Fig. 2 except for a different incident energy of $2 \mathrm{meV}$.
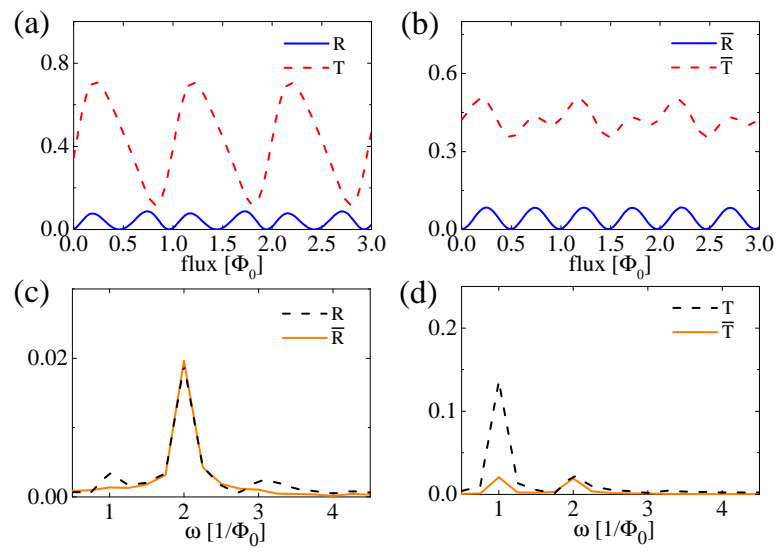

FIG. 6. Oscillation patterns of scattering probabilities and their FFT spectra with disorder strength $5 \mathrm{meV}$. All parameters are the same as those in Fig. 2.

dent energy. The $\Phi_{0} / 2$-period oscillation dominates the return probability $\left(R\right.$ and $\bar{R}$ ) before and after $V_{g}$ averaging. The $\Phi_{0}$-period oscillation of the transmission probability is strongly suppressed by the random $V_{g}$, while the $\Phi_{0} / 2$ component remains unaffected. We also show in Fig. 6 that our results are robust against disorder. Since the main results are stabilized by the time-reversal symmetry, the modification of the sample details will not change the qualitative results. From Figs. 36 6, one can see that the averaged return probability $\bar{R}(\Phi=0)=0$ generally holds for various sample parameters, indicating the universality of the predicted signal of the helical edge states. (a)

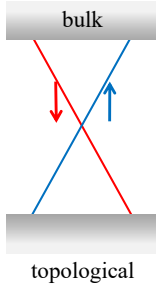

(b)

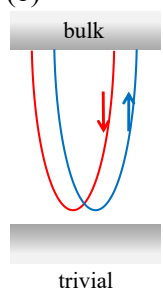

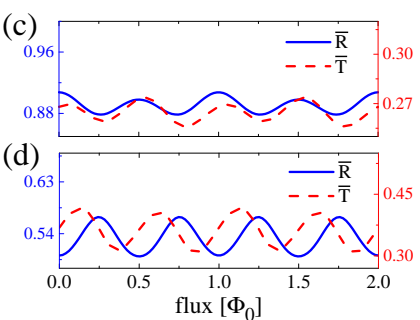

FIG. 7. Sketch of the band structures of (a) topological edge states and (b) trivial edge states. The scattering probabilities of the trivial edge states with (c) $\tilde{\alpha}=0.5 \mathrm{~nm} \mathrm{meV}$ and (d) $\tilde{\alpha}=4 \mathrm{~nm} \mathrm{meV}$.

\section{TRIVIAL EDGE STATES}

Very recently, trivial edge states have been reported in various QSHI candidates, which contribute similar conduction signature to that by the helical edge states [1419. Thus, it is difficult to discriminate the two kinds of edge states with totally different physical origins. The main difference between the helical and trivial edge states can be seen in Fig. 7(a) and 7(b). The helical edge states originate from the band topology of QSHI, which cannot be realized in any one-dimensional nanowire. In contrast, the trivial edge states originate from the surface band bending without topological reasons [15, which resembles those of a nanowire with Rashba spin-orbit coupling [35, as shown in Fig. 7(b). The trivial edge states differ from the helical ones by a doubling of the transport channel, in which backscattering with spin conservation becomes possible. Therefore, the existence of backscattering or not provides a distinctive signal for the identification of helical and trivial edge states, which can be probed in the oscillation pattern of the return probability.

We simulate the trivial edge states by a onedimensional nanowire with Rashba spin-orbit coupling described by the Hamiltonian $H_{\operatorname{Tri}}=k_{x}^{2} /(2 m)+\tilde{\alpha} k_{x} \sigma_{y}$. The AB and AAS effects are studied numerically in the interferometer composed of two nanowires coupling at two points based on the lattice model. A magnetic flux threads the closed loop and a gate voltage is imposed to the upper edge like that in the QSHI model. At the coupling bond between two nanowires, the Rashba spin-orbit coupling $\tilde{\alpha}$ during lateral hopping is included. The oscillation patterns for $\bar{T}$ and $\bar{R}$ after $V_{g}$ averaging are plotted in Figs. 7(c) and 7(d). One can see that the averaged scattering probabilities exhibit a $\Phi_{0} / 2$-period oscillation, indicating an AAS effect dominated scenario. Specifically, for a small Rashba coefficient, the return probability can be captured by $\bar{R}=-\mathcal{R} \sin ^{2}\left(2 \pi \Phi / \Phi_{0}\right)+\mathcal{R}_{0}$; see Fig. 7(c). A small magnetic field results in a decrease of $R$, showing the weak localization effect. For a large Rashba coefficient, the oscillation acquires an extra $\pi$ phase shift, i.e., $\bar{R}=\mathcal{R} \sin ^{2}\left(2 \pi \Phi / \Phi_{0}\right)+\mathcal{R}_{0}$ as shown in Fig. 7(d), which is the weak antilocalization effect. The 
feature of $\bar{R}$ pattern for the trivial edge states provides an effective way to discriminate them from helical edge states. As the oscillation of $\bar{R}$ shows a weak localization behavior as that in Fig. 7(c), trivial edge states are confirmed. Importantly, in both weak localization and antilocalization regimes of the trivial edge states, the return probability is always finite for $\Phi=0$, in start contrast to the helical edge states, thus providing a discriminative signal of the helical and trivial edge states.

\section{DISCUSSION AND SUMMARY}

Some remarks are made below about the experimental implementation of our proposal. The main ingredient of the interferometer is the PC structure introducing interedge coupling which can be achieved by state-of-the-art fabrication techniques 29]. Then two PCs make up an interferometer giving rise to coherent oscillation of the scattering probabilities with varying magnetic field. The distinctive signature of helical and trivial edge states can be extracted from the oscillation pattern of the return probability $\bar{R}$, which is directly related to the differential conductance by $G=\frac{e^{2}}{h}(1-\bar{R})$. Specifically, as the oscillation of $\bar{R}$ exhibits a weak localization scenario, trivial edge states can be identified. If $\bar{R}$ shows a weak antilocalization oscillation, the helical and trivial edge states can be discriminated by zero and finite $\bar{R}$ at $\Phi=0$, respectively. Note that the precondition for such a judgement is the occurrence of coherent oscillation. In our proposal, the absence of return probability should originate from a spin-texture resolved interference effect rather than other trivial physical reasons. The $V_{g}$ averaged return probability $\left.\bar{R}\right|_{\Phi=0}=0$ by AAS interference further reveals the robustness and universality of such a signal, which excludes any accidental result $\left.R\right|_{\Phi=0}=0$ by fine tuning the parameters.

To summarize, we investigate quantum coherent transport through an $\mathrm{AB}$ interferometer constructed by the edge states of a QSHI. By applying a random gate to the edge channel, the $\Phi_{0}$-period $\mathrm{AB}$ oscillation quenches and the $\Phi_{0} / 2$-period AAS oscillation dominates the transport. Such random gate induced AAS oscillation differs from the conventional scenario of disorder averaging. The helical spin texture of topological edge states results in an AAS oscillation of the weak antilocalization type and more importantly the absence of return probability at zero magnetic field. In contrast, the AAS oscillation for the trivial edge states can be of either weak localization or antilocalization type, both having nonzero return probability. Therefore, our proposal provides an effective way for the discrimination between helical and trivial edge states through spin resolved interference effect. Such a signal is protected by time-reversal symmetry, so that it generally holds without fine tuning of the system and is robust against disorder.

\section{ACKNOWLEDGMENTS}

This work was supported by the National Natural Science Foundation of China under Grant No. 12074172 (W.C.), No. 11674160 and No. 11974168 (L.S.), the startup grant at Nanjing University (W.C.), the State Key Program for Basic Researches of China under Grants No. 2017YFA0303203 (D.Y.X.) and the Excellent Programme at Nanjing University.

\section{Appendix A: Lattice model for numerical calculation}

In this Appendix, we elucidate the parameters for the interferometer sketched in Fig. 1(a). The hard-wall boundary in the $y$ direction is described by the following function

$$
|y(x)|= \begin{cases}\left(W-\left(W-W_{P C} e^{\left.-\left(x+L_{M} / 2+L_{P C}\right)^{2} / 30^{2}\right)}\right) / 2,\right. & -L<x<-\left(L_{M} / 2+L_{P C}\right), \\ W_{P C} / 2, & -\left(L_{M} / 2+L_{P C} \leq x \leq-L_{M} / 2,\right. \\ \left(W-\left(W-W_{P C} e^{-\left(x+L_{M} / 2\right)^{2} / 30^{2}}\right)\right) / 2, & -L_{M} / 2<x<0, \\ \left(W-\left(W-W_{P C} e^{-\left(x-L_{M} / 2\right)^{2} / 30^{2}}\right)\right) / 2, & 0 \leq x<L_{M} / 2, \\ W_{P C} / 2, & L_{M} / 2 \leq x \leq\left(L_{M} / 2+L_{P C}\right), \\ \left(W-\left(W-W_{P C} e^{-\left(x-L_{M} / 2-L_{P C}\right)^{2} / 30^{2}}\right)\right) / 2, & \left(L_{M} / 2+L_{P C}\right)<x<L,\end{cases}
$$

where $W$ is the width of the lead, $W_{P C}$ and $L_{P C}$ are the width and length of PC.

The whole devices can be described by Hamiltonian $H=H_{Q S H}+H_{R}$, where $H_{Q S H}$ is the Hamiltonian of QSHI and $H_{R}$ is the Hamiltonian of SOC. Here, we use $\mathrm{HgTe} / \mathrm{CdTe}$ quantum wells in our proposal, which can be described by Bernevig-Hughes-Zhang model as

$$
\begin{aligned}
H_{Q S H} & =-D k^{2}+A k_{x} \tau_{x} \sigma_{z}-A k_{y} \tau_{y}+\left(M-B k^{2}\right) \tau_{z} \\
& =\left(\begin{array}{cccc}
D_{k}+M_{k} & A k_{+} & 0 & 0 \\
A k_{-} & D_{k}-M_{k} & 0 & 0 \\
0 & 0 & D_{k}+M_{k} & -A k_{-} \\
0 & 0 & -A k_{+} & D_{k}-M_{k}
\end{array}\right),
\end{aligned}
$$


where $\sigma_{x, y, z}$ and $\tau_{x, y, z}$ are Pauli matrices of spin and orbital respectively. $k_{ \pm}=k_{x} \pm i k_{y}, k^{2}=k_{x}^{2}+k_{y}^{2}$, $D_{k}=-D k^{2}$, and $M_{k}=M-B k^{2}$. A, B, D and M are the material parameters which can be controlled by experiment. The Hamiltonian of SOC is

$$
\begin{aligned}
H_{R} & =0.5 \alpha\left(1+\tau_{z}\right)\left(k_{x} \sigma_{y}-k_{y} \sigma_{x}\right) \\
& =\left(\begin{array}{cccc}
0 & 0 & -i \alpha k_{-} & 0 \\
0 & 0 & 0 & 0 \\
i \alpha k_{+} & 0 & 0 & 0 \\
0 & 0 & 0 & 0
\end{array}\right)
\end{aligned}
$$

where $\alpha$ is Rashba coefficient.

In order to run numerical calculations, we use a square lattice model for whole system by discretizing the continuous effective hamiltonian $H$. By using $k^{2}=$ $2 a^{-2}\left[2-\cos \left(k_{x} a\right)-\cos \left(k_{y} a\right)\right], k_{x}=a^{-1} \sin \left(k_{x} a\right), k_{y}=$ $a^{-1} \sin \left(k_{y} a\right)$, we can derive the Hamiltonian to real space.
The lattice Hamiltonian is

$$
\begin{array}{r}
H=\sum_{i=1} c_{i}^{\dagger} H_{i i} c_{i}+\sum_{i=1} c_{i}^{\dagger} H_{i, i+a_{x}} c_{i+a_{x}} \\
+\sum_{i=1} c_{i}^{\dagger} H_{i, i+a_{y}} c_{i+a_{y}}+H . C .
\end{array}
$$

where $c_{i}=\left(c_{s, \uparrow, i}, c_{p, \uparrow, i}, c_{s, \downarrow}, i, c_{p, \downarrow, i}\right)$ are the annihilate operators of electron with spin up and spin down in $\mathrm{s}$ and p orbits at site i. The $H_{i i}, H_{i, i+a_{x}}$ and $H_{i, i+a_{y}}$ are $4 \times$ 4 Hamiltonians,

$$
\begin{aligned}
H_{i i} & =-\frac{4 D}{a^{2}}-\frac{4 B}{a^{2}} \tau_{z}+M \tau_{z} \\
H_{i, i+a_{x}} & =\frac{D+B \tau_{z}}{a^{2}}+\frac{A \tau_{x} \sigma_{z}}{2 i a}+\frac{\alpha\left(1+\tau_{z}\right) \sigma_{y}}{4 i a} \\
H_{i, i+a_{y}} & =\frac{D+B \tau_{z}}{a^{2}}+\frac{i A \tau_{y}}{2 a}+\frac{i \alpha\left(1+\tau_{z}\right) \sigma_{x}}{4 a}
\end{aligned}
$$

where lattice constant is $a=3 \mathrm{~nm}$.
[1] X.-L. Qi and S.-C. Zhang, Rev. Mod. Phys. 83, 1057 (2011)

[2] M. Z. Hasan and C. L. Kane, Rev. Mod. Phys. 82, 3045 (2010)

[3] C. L. Kane and E. J. Mele, Phys. Rev. Lett. 95, 226801 (2005)

[4] B. A. Bernevig and S.-C. Zhang, Phys. Rev. Lett. 96, $106802(2006)$

[5] B. A. Bernevig, T. L. Hughes, and S.-C. Zhang, Science 314, 1757 (2006)

[6] C. Liu, T. L. Hughes, X.-L. Qi, K. Wang, and S.-C. Zhang, Phys. Rev. Lett. 100, 236601 (2008)

[7] M. König, S. Wiedmann, C. Brüne, A. Roth, H. Buhmann, L. W. Molenkamp, X.-L. Qi, and S.-C. Zhang, Science 318, 766 (2007)

[8] I. Knez, R.-R. Du, and G. Sullivan, Phys. Rev. Lett. 107, 136603 (2011)

[9] I. Knez, C. T. Rettner, S.-H. Yang, S. S. P. Parkin, L. Du, R.-R. Du, and G. Sullivan, Phys. Rev. Lett. 112, 026602 (2014)

[10] L. Du, I. Knez, G. Sullivan, and R.-R. Du, Phys. Rev. Lett. 114, 096802 (2015).

[11] L. Fu and C. L. Kane, Phys. Rev. Lett. 100, 096407 (2008).

[12] L. Fu and C. L. Kane, Phys. Rev. B 79, 161408 (2009)

[13] A. Y. Kitaev, Physics-Uspekhi 44, 131 (2001).

[14] B.-M. Nguyen, A. A. Kiselev, R. Noah, W. Yi, F. Qu, A. J. A. Beukman, F. K. de Vries, J. van Veen, S. NadjPerge, L. P. Kouwenhoven, M. Kjaergaard, H. J. Suominen, F. Nichele, C. M. Marcus, M. J. Manfra, and M. Sokolich, Phys. Rev. Lett. 117, 077701 (2016).

[15] F. Nichele, H. J. Suominen, M. Kjaergaard, C. M. Marcus, E. Sajadi, J. A. Folk, F. Qu, A. J. A. Beukman, F. K. de Vries, J. van Veen, S. Nadj-Perge, L. P. Kouwenhoven, B.-M. Nguyen, A. A. Kiselev, W. Yi, M. Sokolich, M. J. Manfra, E. M. Spanton, and K. A. Moler, New Journal of Physics 18, 083005 (2016).

[16] S. Mueller, C. Mittag, T. Tschirky, C. Charpentier,
W. Wegscheider, K. Ensslin, and T. Ihn, Phys. Rev. B 96, 075406 (2017)

[17] V. Sazgari, G. Sullivan, and i. d. I. i. d. I. Kaya, Phys. Rev. B 100, 041404 (2019).

[18] F. K. de Vries, T. Timmerman, V. P. Ostroukh, J. van Veen, A. J. A. Beukman, F. Qu, M. Wimmer, B.-M. Nguyen, A. A. Kiselev, W. Yi, M. Sokolich, M. J. Manfra, C. M. Marcus, and L. P. Kouwenhoven, Phys. Rev. Lett. 120, 047702 (2018).

[19] F. K. de Vries, M. L. Sol, S. Gazibegovic, R. L. M. o. h. Veld, S. C. Balk, D. Car, E. P. A. M. Bakkers, L. P. Kouwenhoven, and J. Shen, Phys. Rev. Research 1, 032031 (2019).

[20] C.-Y. Hou, E.-A. Kim, and C. Chamon, Phys. Rev. Lett. 102, $076602(2009)$

[21] T. L. Schmidt, Phys. Rev. Lett. 107, 096602 (2011).

[22] S. Das and S. Rao, Phys. Rev. Lett. 106, 236403 (2011)

[23] A. Soori, S. Das, and S. Rao, Phys. Rev. B 86, 125312 (2012).

[24] J. M. Edge, J. Li, P. Delplace, and M. Büttiker, Phys. Rev. Lett. 110, 246601 (2013)

[25] F. Romeo and R. Citro, Phys. Rev. B 90, 155408 (2014)

[26] W. Chen, W.-Y. Deng, J.-M. Hou, D. N. Shi, L. Sheng, and D. Y. Xing, Phys. Rev. Lett. 117, 076802 (2016).

[27] B. Al'Tshuler, A. Aronov, and B. Spivak, Jetp Lett 33, 94 (1981)

[28] B. Al'tshuler, A. Aronov, B. Spivak, D. Y. Sharvin, and Y. V. Sharvin, JETP Lett 35, 588 (1982).

[29] J. Strunz, J. Wiedenmann, C. Fleckenstein, L. Lunczer, W. Beugeling, V. L. Müller, P. Shekhar, N. T. Ziani, S. Shamim, J. Kleinlein, et al., Nature Physics 16, 83 (2020)

[30] D. Rothe, R. Reinthaler, C. Liu, L. Molenkamp, S. Zhang, and E. Hankiewicz, New Journal of Physics 12, $065012(2010)$.

[31] A. Ström, H. Johannesson, and G. Japaridze, Physical review letters 104, 256804 (2010).

[32] J. I. Väyrynen and T. Ojanen, Physical review letters 
106, 076803 (2011)

[33] J. H. Bardarson and J. E. Moore, Reports on Progress in Physics 76, 056501 (2013).

[34] C. W. Groth, M. Wimmer, A. R. Akhmerov, and
X. Waintal, New Journal of Physics 16, 063065 (2014).

[35] T. Haidekker Galambos, S. Hoffman, P. Recher, J. Klinovaja, and D. Loss, Phys. Rev. Lett. 125, 157701 (2020) 